\title{
Quantification of Fetal ST-Segment Deviations
}

\author{
Angela Agostinelli ${ }^{1}$, Mariachiara Di Cosmo ${ }^{1}$, Agnese Sbrollini ${ }^{1}$, Luca Burattini ${ }^{2}$, Micaela Morettini ${ }^{1}$, \\ Francesco Di Nardo ${ }^{1}$, Sandro Fioretti ${ }^{1}$, Laura Burattini ${ }^{1 *}$ \\ ${ }^{1}$ Department of Information Engineering, Università Politecnica delle Marche, Ancona, Italy \\ ${ }^{2}$ Department of Clinical Sciences, Università Politecnica delle Marche, Ancona, Italy
}

\begin{abstract}
By fetal electrocardiogram (FECG) analysis it has been found that changes in the ST segment are associated with acid-base status, and thus fetus health state. Currently, the most popular estimation of fetal STsegment deviations is performed as ratio between T-wave height and QRS-complex amplitude using the STAN monitor. Thus, this evaluation is indirect because not directly derived from measurements on the ST segment. This study proposes a new procedure for an automated direct quantification of fetal ST-segment deviations, which are described in terms of ST-amplitude and STtrend. Particularly, ST-amplitude corresponds to the maximum of the mean amplitude values obtained through a moving-average $(15 \mathrm{~ms})$ operation over the $S T$ segment. Instead, ST-trend corresponds to the difference between the ST-segment amplitudes calculated in the first and the last of three intervals in which the ST segment is divided; thus, ST-trend sign indicates a ST-segment elevation (positive sign) or depression (negative sign). The procedure was evaluated on five direct FECG recordings (in https://physionet.org/physiobank/database/adfecgdb/). Mean values (over population) of ST-amplitude and STtrend were $9.6 \pm 5.5 \mu \mathrm{V}$ and $1.4 \pm 2.3 \mu \mathrm{V}$, respectively. All found values were validated by visual inspection of the magnified FECG plots.
\end{abstract}

\section{Introduction}

Fetal surveillance during pregnancy, and in particular during labor, enables identification of fetuses at risk for neonatal acidosis. Electronic fetal monitoring started with the introduction of cardiotocography (around 1960), a test that provides information on the fetus health state on basis of visual interpretation of the fetal heart-rate tracing [1]. Despite its widespread use, visual interpretation of cardiotocographic tracings is associated with limited reliability [2]. Combination of cardiotocography with fetal electrocardiography (FECG) could be helpful to prevent fatal perinatal outcomes $[3,4]$.
FECG is a recording of the electrical activity of the fetal heart that can be either direct or indirect. Direct FECG is acquired by positioning an electrode on the fetal scalp during labor. Instead, indirect FECG is acquired by positioning electrodes on the mother's abdomen during late pregnancy and labor. Direct FECG is invasive but characterized by a higher signal quality than indirect FECG, which is noninvasive but highly corrupted by noise of various kinds [3]. Independently by its acquisition modality, FECG morphology is very much related to adult electrocardiography since containing the same basic waveforms: $\mathrm{P}$ wave, associated to atrial depolarization; QRS complex, associated to ventricular depolarization; and $\mathrm{T}$ wave (and $\mathrm{U}$ wave, when present), associated to ventricular repolarization. In addition to heart-rate monitoring, FECG allows clinical evaluations strictly related to its morphology [2]. Specifically, P-wave and QRS-complex duration are used to evaluate the intrauterine growth restriction [6]; widened and biphasic QRS complexes not preceded by $\mathrm{P}$ waves (which manifest when supraventricular extrasystoles occur) indicate cases of congenital heart diseases [7]; eventually, changes of ST segment (which connects QRS complex and $\mathrm{T}$ wave, and reflects the effect of fetal myocardial hypoxia) are correlated with acid-base status [3].

FECG has been studied for many decades, but only in the last thirty years computerized waveforms analysis has led to its clinical use [8,9]. For what concerns STsegment evaluation, it is currently performed by quantifying fetal ST-segment deviations as the ratio between T-wave height and QRS-complex amplitude (T/QRS ratio) using the STAN monitor (Neoventa Medical, Göteborg, Sweden) [2]. When the T/QRS ratio exceeds a certain threshold (0.30), ST-segment elevation is detected and an ST event is flagged on the STAN monitor $[8,9]$. This evaluation is indirect because not directly derived from measurements on the ST segment. Alternatively, this study proposes a new procedure for an automated direct quantification of fetal ST-segment deviations, which are described in terms of ST-segment amplitude and trend. 


\section{Clinical Data and Methods}

\subsection{Clinical Data}

Clinical data consist of five FECG recordings; each (60 s long), acquired from five different pregnant women during labor, which occurred within the $38^{\text {th }}$ to $41^{\text {st }}$ week of gestation). Specifically, during labor, when the membranes were ruptured and the uterine cervix was dilated, a scalp electrode was applied to the fetal head and FECG was recorded [5]. All acquisitions (sampling frequency: $1000 \mathrm{~Hz}$ ) were performed at the Department of Obstetrics at the Medical University of Silesia, and are part of "Abdominal and Direct Fetal Electrocardiogram Database" [10] of PhysioNet (www.physionet.org) [11], freely accessible on the web under the ODC Public Domain Dedication and License v1.0. The database has been fully anonymized and may be used without further Institutional Review Boards approval.

\subsection{Automated quantification of fetal ST- segment deviations}

Each FECG was preprocessed before being submitted to our automated algorithm for ST-deviations quantification. Preprocessing consisted $200 \mathrm{~Hz}$ downsampling, bandpass filtering $(0.5-45 \mathrm{~Hz})$, R-peak identification [12] and baseline removal. Successively, ST-segment deviations were quantified. Our automated quantification of ST-segment deviations consists of the following four steps: 1) FECG median-beat computation (from which ST-segment deviations are measured); 2) Jpoint localization; 3) ST-segment identification; and 4) quantification of ST-segment deviations. The block diagram of the algorithm is reported in Fig. 1 whereas the description of each step is reported below.

1) FECG median-beat computation. After application of our fetal R-peak detection algorithm [12], FECG is filtered for noise reduction by applying the Segmented-Beat Modulation Method (SBMM) [5,13,14], Successively, the median (for further noise reduction) beat is computed in representation of the FECG morphology.

2) J-point localization. J point represents the junction between QRS complex and ST segment, and thus, is considered the ST-segment beginning. In this fetal application, its localization is identified $35 \mathrm{~ms}$ after the $\mathrm{R}$ peak (Fig.2).

3) ST-segment identification. ST segment is the signal segment between the QRS complex and $\mathrm{T}$ wave. Thus, ST-segment identification starts from J point and ends $60 \mathrm{~ms}$ after $\mathrm{J}$ point (Fig.2). Consequently, in this study the ST segment is always considered $60 \mathrm{~ms}$ long.

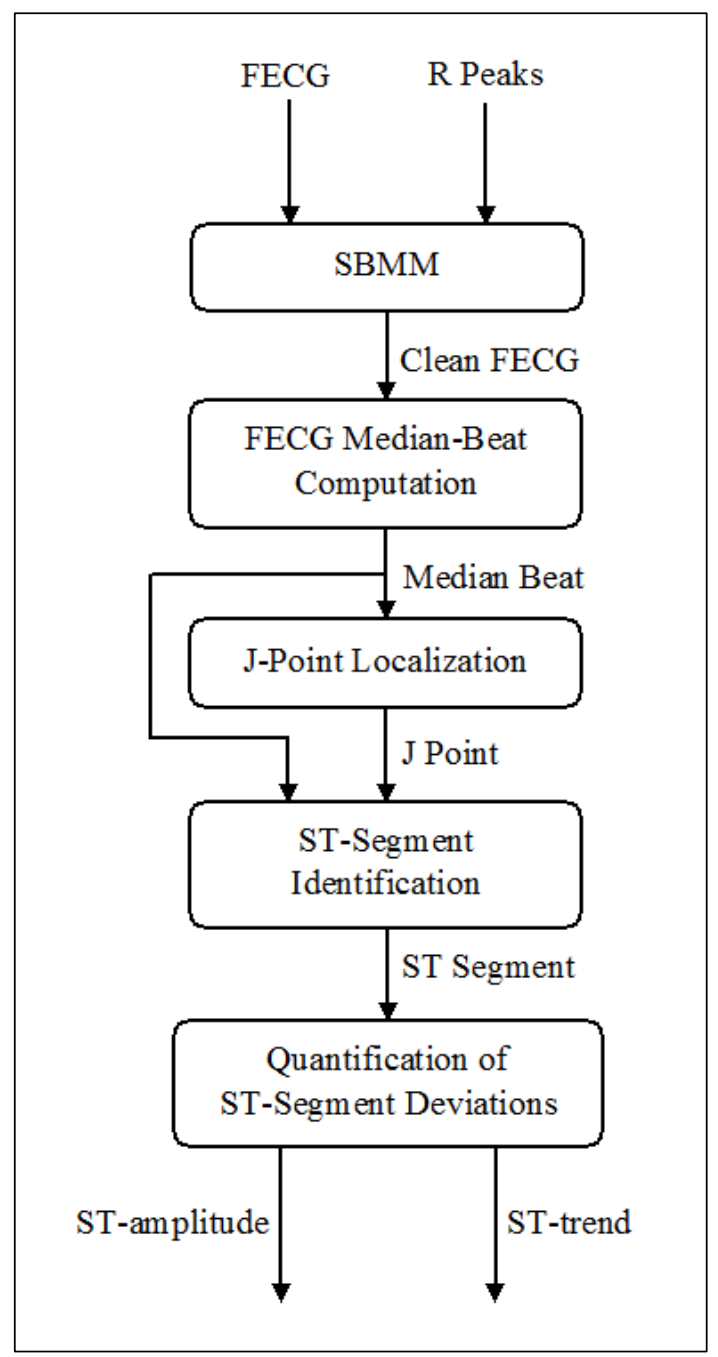

Fig.1. Block diagram of the algorithm for ST-deviations quantification.

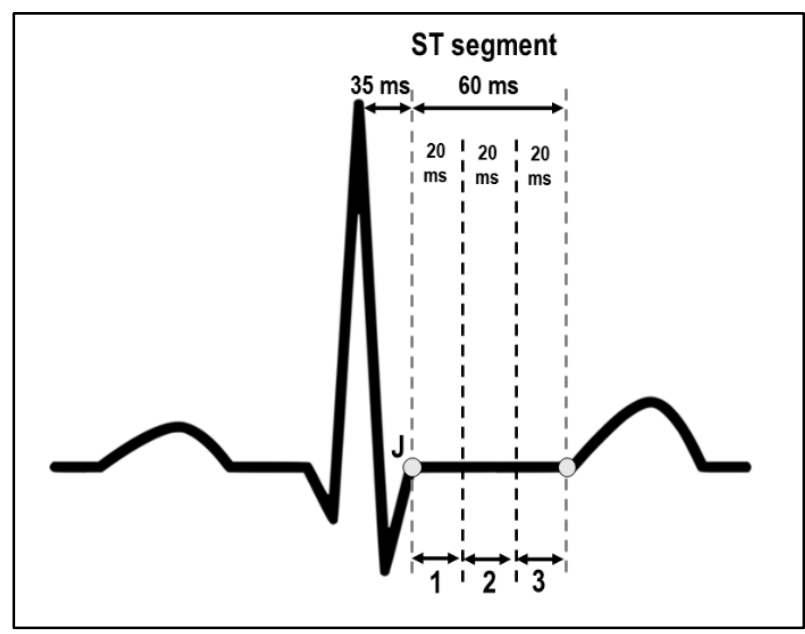

Fig. 2. Identification of ST-segment and three intervals in which the segment is divided. 
4) Quantification of ST-segment deviations. Quantification of ST-segment deviations was quantified by means of two parameters: ST-amplitude and ST-trend. ST-amplitude corresponds to the maximum of the mean amplitude values obtained through a moving-average (15 ms) operation over the ST segment. Instead, ST trend corresponds to the difference between the ST-segment amplitudes calculated in the first and the last of three intervals (I1, I2, and I3; each $20 \mathrm{~ms}$ long) in which the ST segment is divided [15] (Fig 2). Thus, the sign of STtrend indicates a ST-segment elevation (positive sign) or depression (negative sign).

\subsection{Statistics}

Each FECG tracing was characterized by a value of ST-amplitude and ST-trend. Distribution of these two parameters over the population was described in terms of mean \pm standard deviations.

\section{Results}

The ST-amplitude and ST-trend values relative to each FECG are reported in Table 1. On average, ST-amplitude was quite low $(9.6 \pm 5.5 \mu \mathrm{V})$; in two cases (FECG2 and FECG3) it reached $15 \mu \mathrm{V}$. ST-trend was 0 in one case (FECG1), positive in three cases (FECG2, FECG3 and FECG5) and negative in one case (FECG4); on average, it was $1.4 \pm 2.3 \mu \mathrm{V}$. To evaluate goodness of STdeviations quantification, results relative to all tracings have been validated by visual inspection of the magnified FECG plots, which are reported in Fig. 3. As it can be seen, absence of ST-deviations relative to FECG1 is easily visually confirmed. Also, ST-deviations relative to FECG2 and FECG3 (relatively high ST-amplitude and positive ST-trend) are visually recognizable. Instead, STdeviations relative to FECG4 and FECG5 (depression and elevation, respectively) are hardly detectable by the naked eye, since associated to low values of ST-amplitude.

Table 1. ST-deviations characterization in terms of ST amplitude and ST-trend relative to each of the 5 FECG recordings in the study database.

\begin{tabular}{lcc}
\hline $\begin{array}{l}\text { FECG } \\
\text { recordings }\end{array}$ & $\begin{array}{c}\text { ST-amplitude } \\
(\mu \mathrm{V})\end{array}$ & $\begin{array}{c}\text { ST-trend } \\
(\mu \mathrm{V})\end{array}$ \\
\hline FECG1 & 3 & 0 \\
FECG2 & 15 & 3 \\
FECG3 & 15 & 4 \\
FECG4 & 6 & -2 \\
FECG5 & 9 & 2 \\
\hline
\end{tabular}
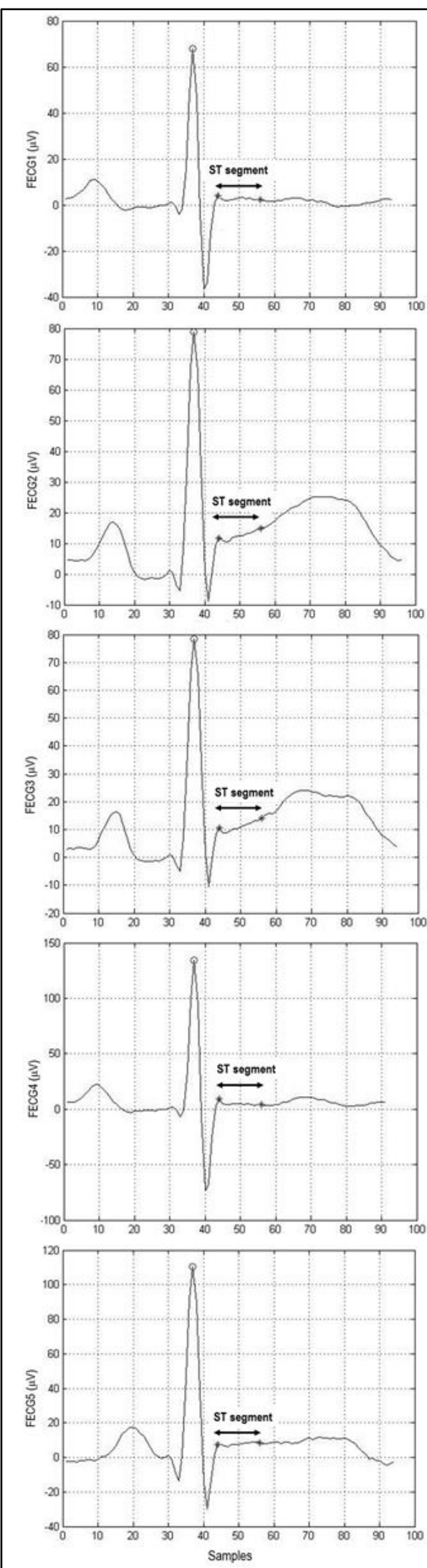

Fig. 3. Magnified plots relative to each FECG signal. 


\section{Discussion}

This study proposes a new procedure for an automated quantification of fetal ST-segment deviations. Differently from the STAN algorithm, this procedure provides an evaluation directly derived from measurements on the ST segment, and describes ST deviations by means of two innovative parameters: ST-amplitude and ST-trend, respectively indicating ST-deviations amplitude and slope. To provide reliable results, ST deviations should be quantified in FECG signals of good quality. Thus, FECG tracings, beside preprocessing, were further filtered for noise removal by our previously proposed SBMM [13], which proved to be particularly robust to noise $[13,14]$. Successively, the median beat was computed for further noise reduction and for a brief (only two parameter values, those relative to ST-amplitude and ST-trend) characterization of the ST-segment deviations occurring in a minute-long FECG tracing. Still, in good quality FECG the proposed procedure for characterization of STsegment deviations may also be applied to each single heartbeat in the signal, allowing, for example, evaluation of the ST-segment deviations variability. Identification of ST-segment onset and offset was performed considering fixed time intervals after the R peak $(35 \mathrm{~ms}$ and $95 \mathrm{~ms}$, respectively, so that ST-segment is always $60 \mathrm{~ms}$ long). This choice was taken after the experimental finding that the identification error derived from the application of automated procedures for ST-segment endpoints identification is of the same order of magnitude (if not greater) of the error derived by using fixed interval.

The number of FECG tracings in the database used in this methodological study is too little to allow significant statistical evaluations on the populations. Moreover, unfortunately no clinical report relative to the fetus conditions were available in the used "Abdominal and Direct Fetal Electrocardiogram Database" [10], so that not possible to link the obtained results to acid-base status of the fetuses in order to evaluate the metabolic acidosis birth. Nevertheless, some general important methodological information can be derived from the results. Specifically, ST-segment deviations have to be characterized by a sufficiently high value of T-amplitude (greater than $10 \mu \mathrm{V}$ ), independently by the sign of STtrend (Fig.3, plots relative to FECG4 and FECG5). Eventually, marked ST-segment deviations are characterized by high absolute values of both parameters.

Future studies are need to further validate the proposed algorithm for ST-segment deviations characterization. Such studies will involve larger populations with known fetal outcomes to evaluate the method ability to identify fetuses at risk for neonatal acidosis; and use indirect FECG, from which ST-deviations characterization is more challenging but clinically more relevant.

\section{References}

[1] Steer PJ. Fetal distress. Curr Obstet Gynaecol 2002;12:1521.

[2] Dervaitis KL, Poole M, Schmidt G, et al. ST segment analysis of the fetal electrocardiogram plus electronic fetal heart rate monitoring in labor and its relationship to umbilical cord arterial blood gases. Am J Obstet Gynecol 2004;191:879-884.

[3] Agostinelli A, Grillo M, Biagini A, et al. Noninvasive fetal electrocardiography: an overview of the signal electrophysiological meaning, recording procedures, and processing techniques. Ann Noninvasive Electrocardiol. 2015;20:303-313.

[4] Vayssiere C, Haberstich R, Sebahoun V, et al. Fetal electrocardiogram ST-segment analysis and prediction of neonatal acidosis. Int J Gynecol Obstet 2007;97:110-114.

[5] Agostinelli A, Sbrollini A, Burattini L, et al. Noninvasive Fetal Electrocardiography Part II: Segmented-Beat Modulation Method for Signal Denoising. TOBEJ 2017 (ahead of print).

[6] Baschat AA. Integrated fetal testing in growth restriction: combining multivessel Doppler and biophysical parameters. Ultrasound Obstet Gynecol 2003;21:1-8.

[7] Hornberger LK, Sahn DJ. Rhythm abnormalities of the fetus. Heart 2007;93:1294-1300.

[8] Rose KG, Amer-Wahlin I, Luzietti R, et al. Fetal ECG waveform analysis. Best Prac Res Clin Obstet Gynaecol 2004; 18:485-514.

[9] Amer-Wahlin I, Arulkumaran S, Hagberg H, et al. Fetal electrocardiogram: ST waveform analysis in intrapartum surveillance. Int J Gynecol Obstet 2007;114:1191-1193.

[10] Jezewski J, Matonia A, Kupka T, et al. Determination of fetal heart rate from abdominal signals: evaluation of beatto-beat accuracy in relation to the direct fetal electrocardiogram. Biomed Tech 2012;57:383-394.

[11] Goldberger AL, Amaral LA, Glass L, et al. PhysioBank, PhysioToolkit, and PhysioNet: components of a new research resource for complex physiologic signals. Circulation 2000;101:E215-E220.

[12] Agostinelli A, Marcantoni I, Moretti E, et al. Noninvasive Fetal Electrocardiography Part I: Pan-Tompkins' Algorithm Adaptation to Fetal R-peak Identification. TOBEJ 2017 (ahead of print).

[13] Agostinelli A, Giuliani C, Burattini L. Extracting a clean ECG from a noisy recording: a new method based on segmented-beat modulation. Computing In Cardiology 2014;41:49-52.

[14] Agostinelli A, Sbrollini A, Giuliani C, et al. Segmented Beat Modulation Method for Electrocardiogram Estimation from Noisy Recordings. Med Eng Phys 2016;38:560-568.

[15] Faganeli, J, Jager F. Automatic Distinguishing Between Ischemic and Heart-Rate Related Transient ST Segment Episodes in Ambulatory ECG Records. Computers in Cardiology 2008;35:381-384.

Address for correspondence.

Laura Burattini

DII, Università Politecnica delle Marche,

via Brecce Bianche 12, 60131, Ancona, Italy.

E-mail address. 1.burattini@univpm.it. 